\title{
On the Universality of the Quantum Approach
}

\author{
Maria Kuman* \\ Holistic Research Institute, USA \\ *Corresponding author: Maria Kuman, Holistic Research Institute, 1414 Barcelona Dr., Knoxville, TN 37923, USA.
}

Submission: 眥 January 10, 2019; Published: 海 February 11, 2019

\begin{abstract}
In the quantum world everything is interconnected one energy system. If so, quantum mechanics cannot be applicable only to the micro-world if the macro-world is built by micro-particles. At least now when we built quantum computers. We must acknowledge the fact that quantum mechanics must apply to more than the micro-world. Our studies lead to the same conclusion. In the way elementary particles emit and suck back virtual photons the active Sun emits spinning plasma balls which are sucked back. Thus, the micro- and macro-world exhibit the same dynamics and have the same torusshaped nonlinear electromagnetic fields (NEMF). Also, helium nano-droplets exhibit at very low temperatures the same turbulent dynamics and have the same torus-shaped NEMF. It seems that quantum behavior is specific for all spinning NEMF exhibiting turbulence regardless are those elementary particles from the micro-world or sun (stars) from the macro-world. Russian measurements sowed that our human NEMF is also spinning, has a torus shape and has active cycles with exhibited turbulence. If we embrace the fact that the whole world is quantum and interconnected, we could easily explain the influence of our mind on the quantum experiments we do.
\end{abstract}

Keywords: Physics; Nonlinear physics; Universal quantum approach; Turbulence and quantum levels; Human quantum energy levels

\section{Introduction}

Let us introduce some concepts of nonlinear physics, which we would need. The flux of running river-water would be linear, if the bottom of the river is smooth. However, if there is a big stone on the bottom of the river, the water needs to flow around the stone and the water flux becomes nonlinear. Behind the stones, turbulence would be observed manifested with a couple of vortex spinning clockwise and anti-vortex spinning counterclockwise.

Following the law of the folded fingers of the right hand in physics, when the folded fingers show the direction of the currents, the vertical thumb show the direction of the induced magnetic field. Following this law, the vortices (which spin clockwise) would induce magnetic field toward the surface. This would make the vortices to suck energy in. Following the same law the anti-vortices (which spin counterclockwise) would induce magnetic field off the surface, which would make the anti-vortices to emit energy.

Ilya Prigogine, who took Nobel Prize in 1977 for his nonlinear nonequilibrium theory describing the dynamic of self-organized systems, writes in his book Time, Dynamics, and Chaos [1] that the final state of development or evolution of a dynamic system is called attractor. There are four different types of attractors, onedimensional (point) attractor, two-dimensional (cyclic) attractor, three-dimensional (torus) attractor, and four-dimensional (strange) attractor. They are all self-organized systems, which are very sensitive to initial and boundary conditions.
These attractors are the final states of development of real dynamic systems with energy dissipation, which evolved in perfect order out of the chaos. In this article the thee-dimensional torus attractor will be at the center of our attention (Figure 1). The author published a generalized theory of the attractors in [3].

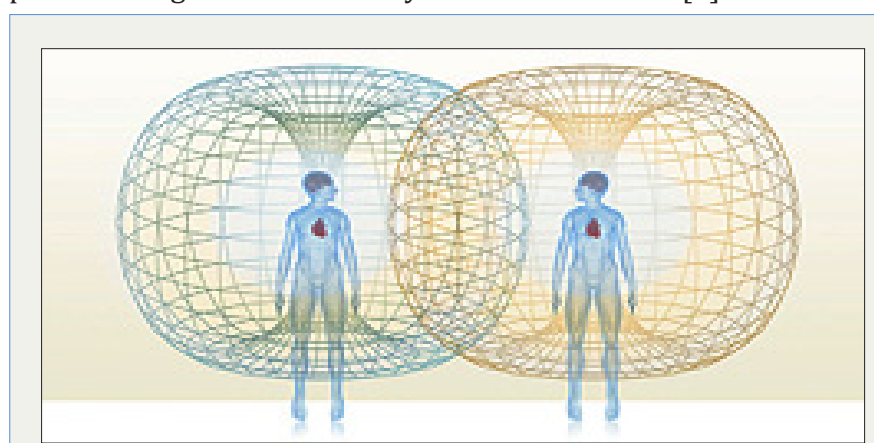

Figure 1: The donut shaped human NEMF of the heart as measured by the heart math Institute in California [2] (adopted from their webpage).

What We Can Learn from the Russian Measurements about the Spinning of the Human NEMF

The Russian scientist Shkatov patented equipment, called 'torsemeter', which allows him to measure the spinning of the human NEMF $[4,5]$. With the torsemeter he measured how positive emotions (joy, happiness...) increase the spinning of the human's 
NEMF in positive (clockwise) direction and how negative emotions (anger, hatred, jealousy...) increase the spinning of the human NEMF in negative (counterclockwise) direction [4].

Since in nonlinear physics turbulent spinning in clockwise direction is called vortex and vortices suck energy, energy must be sucked into our NEMF during positive emotions, which would explain the uplifting we feel when experiencing positive emotions. Since in nonlinear physics turbulent spinning in counterclockwise direction is called anti-vortex and anti-vortices emit energy must be emitted from our NEMF during negative emotions, which would explain the energy drop we feel when experiencing negative emotions [4].

\section{Our Measurements of the Human's NEMF}

This agrees completely with the measurements on people done with our patented supersensitive meter measuring millionth of the ampere and less.
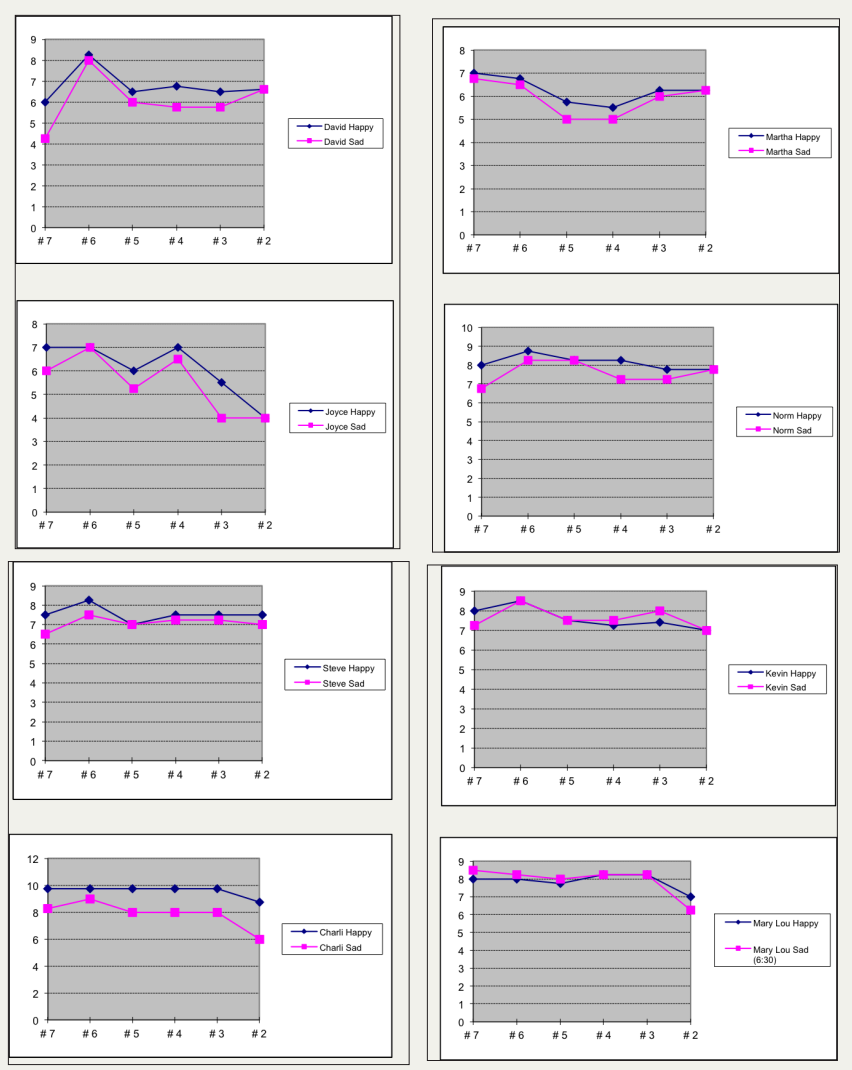

1. We asked people to think about the happiest moments of their life and we measured their energy. We found that positive thoughts increase their energy (blue curves on Figure 2). We also found that positive thoughts improve the energy balance by filling with more energy the places with lowest energy (just like a river would fill with more water the lowest places). This improved energy balance means improved health. Therefore, positive thoughts make you healthier (Figure 2) [6].

2. We asked people to think about the saddest (most unhappy) moments of their life and we measured their energy. We found that negative thoughts not only decrease the body energy, they worsen the energy balance (pink curves on Figure 2). When speaking with the measured people, it became obvious that what was dropping in energy maximum was their genetically inherited weak organ with lowest energy. This means that each negative thought takes you a step farther to a disease of your genetically inherited weak organ. In other words negative thoughts make you sicker (Figure 2) [6].

Figure 2: The body energy balance measured at the alternating vortices and anti-vortices of the human NEMF.

The measurements with our patented sensitive energy meter showed that:

1. Positive emotions (or just positive thinking) increase the human energy and improves their energy balance (blue curves), which makes them healthier.

2. Negative emotions (or just negative thinking) decrease the human energy and worsen the energy balance (purple curves), which is a step toward a disease.
When David was sad (negative thinking), his heart energy dropped maximum and he said that both of his parents died of heart attack. The same was true for Norm, who said that his father was a negative thinker and died of heart attack. When Joyce was sad, her stomach energy dropped maximum and she said she know that her stomach is her weak place because each time she gets upset, her stomach gets upset.

When Charlie was sad his prostate energy dropped maximum, but he didn't have any complains yet. However, I met Charlie ten 
years after I did the measurements and asked him how his prostate is. He smiled and said: "Gone for good." I asked him what this means. He said: "It started creating troubles and they removed it."

When Martha was sad her Thyroid Gland energy dropped maximum and she said her mother had thyroid problem. So, it became obvious that when the people were sad or upset the energy of their genetically inherited weak organ was maximally dropping. This is in full agreement with the explanation of Selye that the same stress causes different diseases in different individuals because just as in engineering stress cracks a material at the weakest place, psychological stress cracks the weakest place of the body the genetically inherited weak organ which is different for different individuals.

Reiki healers and their patients were also measured with our supersensitive equipment (Figure 3). Reiki healing is energy healing done by holding hands over the bodies of the patients. 'Rei-ki' means 'universal energy' and our measurements showed that indeed universal energy must be involved because after Reiki healing not only was the energy of the patient higher and more balanced, the energy of the healer was higher and more balanced, which is possible only if a third source of energy is involved (Figure 3) [7].
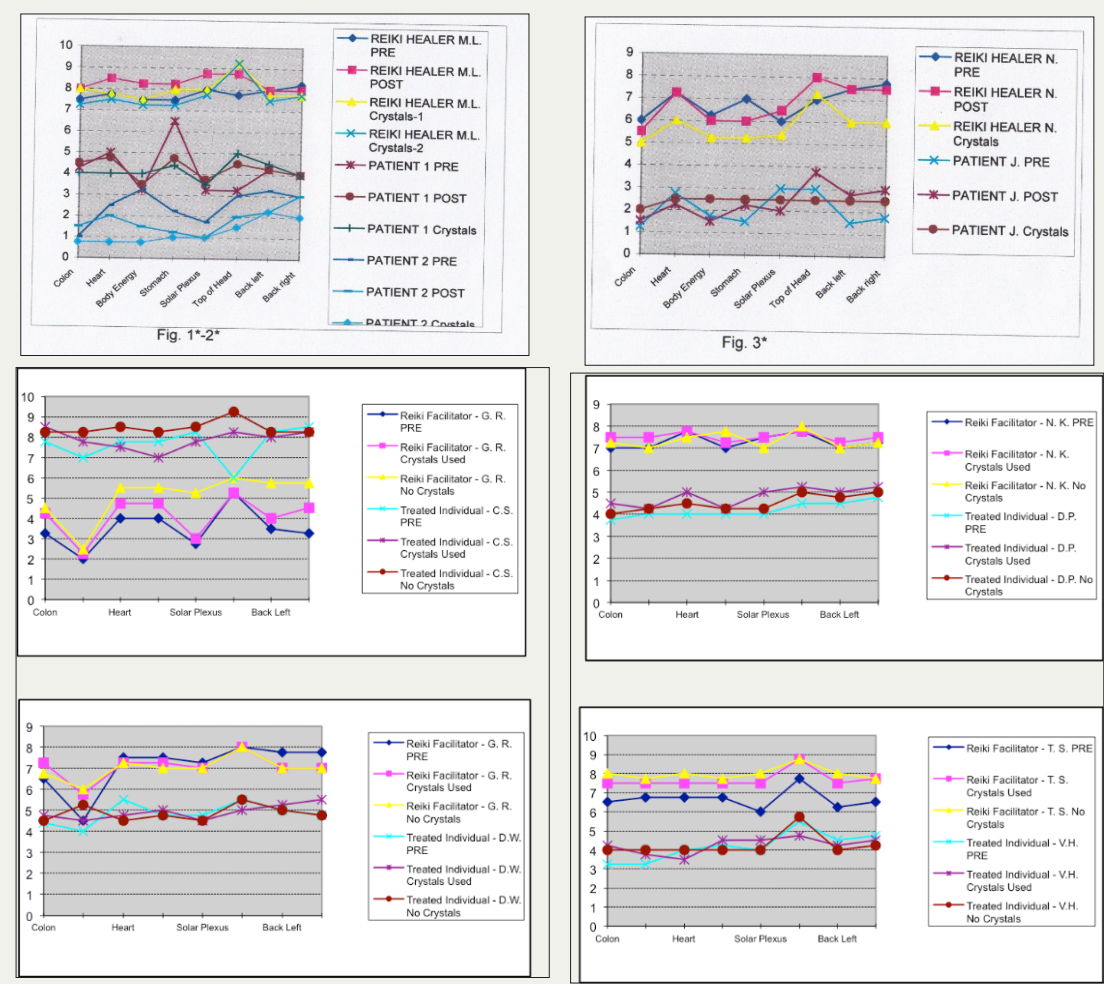

Figure 3: Top curves - the energy of the Reiki Healers before (PRE) and after (POST) Reiki Healing. Lower curves -the energy of the treated people before (PRE) and after (POST) Reiki healing.

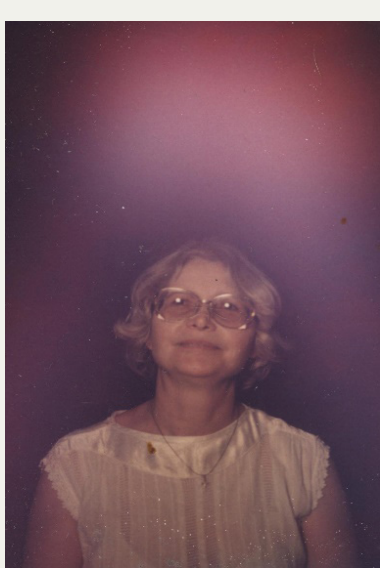

Figure 4: Kirlian photography of the author in 1991-the light ball means quantum level 9.

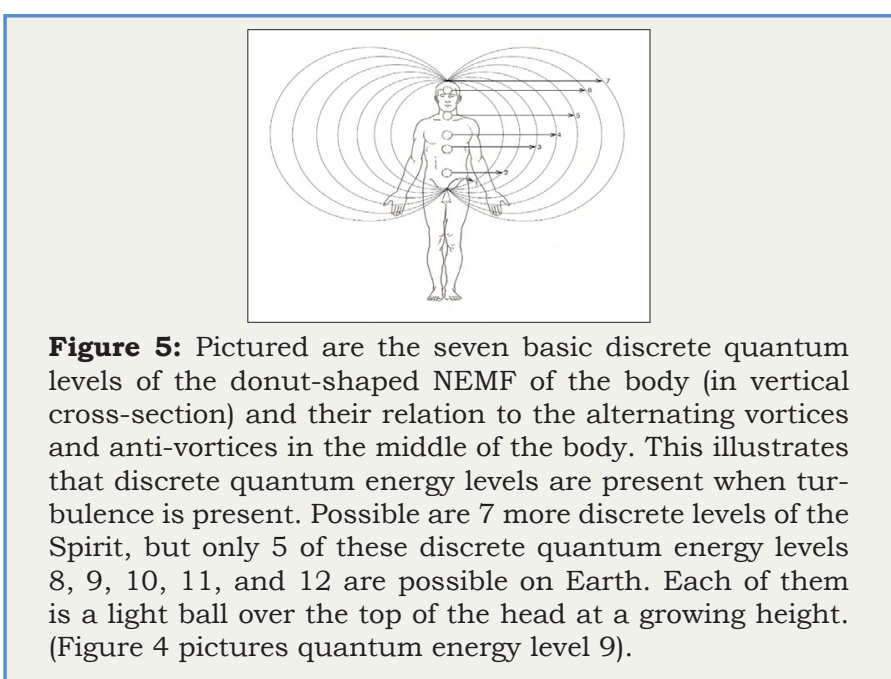


We also noticed that after each Reiki healing the energy of the healer was higher on the top of his head at the acupuncture point Bai Huei (Point of Union). This point is listed on the horizontal axis of (Figure 3) between the points of Solar Plexus and Back Left. The energy increase at point Bai Huei indicates that the top of the head was the portal, through which the Universal energy was entering the body of the healer (Figure $4 \& 5$ ).

Since the hole of the donut-shaped NEMF starts at the top of the head and finishes at the tailbone (Figure 5). Which is the axis of spinning of the donut, obviously the Reiki Healers suck electrical energy from the atmosphere (Universal energy) through the top of their head where the opening of their donut shaped NEMF is $[6,7]$.

The frequency of the electric field of the atmosphere was $8 \mathrm{~Hz}$ and the measured frequency of the energy emitted from the hands of Reiki Healers was found to be 5 to $20 \mathrm{~Hz}$ in Japan [8] and 0.3 to $30 \mathrm{~Hz}$ in the US [9]. Which includes the frequency $8 \mathrm{~Hz}$. Therefore, the Reiki Healers draw electrical energy from the atmosphere through the top of their heads.

To check how the energy received by the Reiki Healers through the tops of their heads, is delivered through their hands to the patients, Reiki Healers were asked to hold one hand on the vortex Solar Plexus (one inch under the ribs conjunction in front of the body). While healing with the other hand. We found that energy was not delivered to the patient.

However, energy was delivered if the hand, instead of being on the Solar Plexus, was hanging down. Therefore, the energy sucked through the top opening of the donut shaped NEMF of the Reiki Healers is delivered to their hands and to the patients through their vortex Solar Plexus (which is in the middle of the donut axis of spinning) (Figure 5).

The Russian Measurements when Combined with our High-sensitivity Electrical Measurements Reveal the Dynamic of the Human NEMF

Let us combine the measurements done with our patented supersensitive meter with the spinning measurements of Dr. Shkatov in Russia done with his patented torsemeter [10]. The comparison reveals the following dynamic of the human NEMF.

1. Positive thinking and positive emotions make the human NEMF spin faster in clockwise direction. Following the rule of the folded fingers of the right hand at clockwise spinning the vortex point on top of the head (the top hole of the donut-shaped NEMF) would suck energy in. This explains the measured by us increase of energy at positive thinking (especially on top of the head) and also the uplifting we feel at positive emotions or just positive thinking.

When we are happy we have the feeling that time runs faster. Since happy thoughts or feelings make the donut shaped NEMF spin faster this faster spinning obviously creates the perception of faster running time. When Einstein was asked what relativistic time is his joking answer was if you sit and chat with a young beautiful girl two hours can feel as 2 minutes but if you have to sit two minutes on a hot stove two minutes can feel like two hours.

Here is our serious explanation of the joking answer of Einstein. When we feel uplifted or happy two hours can feel as two minutes because our donut shaped NEMF spin clockwise faster which creates the perception of fast running time. Also, the faster spinning of the donut makes energy to be sucked through the hole of the donut on top of the head, which determines our feeling of being uplifted when happy.

2. Negative thinking and negative emotions make the donutshaped human NEMF spin faster counterclockwise. The spinning is around the axis passing through the hole of the donut. Which is along the backbone (Figure 5). Following the rule of the folded fingers of the right hand at counterclockwise spinning the antivortex point on top of the head (the top hole of the donut-shaped NEMF) would emit energy out.

In other words, at negative emotions or just negative thinking the counterclockwise spinning anti-vortex on top of the head would emit energy. This explains our feeling of low energy at negative emotions or just negative thinking. I have seen Kirlian photos (in Russian books) of angry people on which one can see energy emitted from the top of the head like lightening.

\section{Kirlian Photography of the Human Nonlinear Electro- magnetic Field}

The human NEMF has discrete energy levels just like the atoms of which our material body consists. Can we present a proof for this? The human aura is very weak but through the use of high frequency electric field which multiplies manifold the light of our weak aura its photographing can be made possible.

The photographies are named after the Ukrainian scientist Kirlian who we think was the 'first' to start photographing the light of the human aura. The Kirlian photography became very popular in the 1960s [11]. However, almost 100 years before Kirlian the German Baron Karl von Reichenbach first observed and photographed the human light in 1861 [12].

Baron von Reichenbach (born in 1788 in Stuttgart) called the body light 'od' energy from Wodin='All Transcending One'. It meant that the light energy was all penetrating which in the terminology of modern nonlinear physics means nonlinear light energy.

Baron von Reichenbach called the photos 'odographs'. Unfortunately, the achievements of Baron von Reichrenbach were crossed out with the crossing of Mesmerism. We refused to believe the claims of Mesmer that he can heal with magnets just as we refused to believe that the human body can shine and this light can be photographed.

But the words of the Baron must not be forgotten: "Everything emits LIGHT...everything...everything. We live in a world full of SHINING MATTER." Fechner said after Baron von Reichenbach passed away: "The world we sit in would be dark and cold, if we will 
not open our eyes inward to the Spirit and to the internal flame of Nature".

Our nonlinear electromagnetic field has six spinning energy centers along the backbone, which is the axis of spinning of the donut-shape NEMF. They alternate vortex-anti-vortex-vortex-etc. The seventh spinning energy center on top of the head sums up the energy of the six spinning centers under it (Figure 5).

This is because each of the spinning energy centers has its own donut in the aura but all six donuts with different radius are connected in the upper (top of the head) and lower (tailbone) points of the axis of spinning, which are the northern and southern pole of the magnet induced by the spinning NEMF (Figure 5).

Stars and Humans have NEMFs with the Same Torus Shape and Dynamic

Amazingly, in astronomy stars exhibit similar dynamic of sucking or emitting energy through the hole of their donut shaped NEMF. In double stars, it was observed how the dimmer star was sucking energy from the brighter star through the hole of its donut-shaped NEMF, while the brighter star was losing energy also through the hole of its donut. This was continuing until the energies of both stars became equal $[13,14]$.

Recently, Sarafina Nance of the University of Texas at Austin claimed in an article published in Monthly Notices of the Royal Astronomical Society that the rapid spinning of the famous star Betelgeuse was a result of swallowing another star [7]. The swallowed star was probably sucked through the hole of the donut shaped NEMF of Betelgeuse.

Thus, measurements with our patented super-sensitive energy meter showed that just like the stars humans at positive emotions or just positive thinking can suck energy through the top of their head (point 7 on Figure 5). Where the hole of their donut shaped NEMF is. The sucked energy makes the donut shaped NEMF to spin faster clockwise.

\section{What Can We Learn from the Research on Helium Na- nodroplets?}

Not only are our Sun and the other stars containing helium and spinning, helium nanodroplets spin and seem to exhibit behavior similar to the Sun and the stars [3]. In the November's journal of Physics Today, 2014, p. 16, Ashley Smart wrote the article Quantized Vortices in a Nanodroplet [15], in which she describes the unusual behavior observed in super-fluid fast-spinning helium nanodroplets at temperatures close to the absolute zero.

The marked circles on (Figure 6a) present the peaks of neutron scattering from helium nanodroplets, which mean quantum behavior. Analysis showed that the droplets were donut shaped and they were belched at the equator, which meant they were spinning fast clockwise and sucking energy. The increased spinning resulted in increased turbulence in their equatorial area manifested with a chain of alternating vortices and anti-vortices, which exhibited quantum behavior and led to the observed peaks of Bragg's neutron scattering.

However, the helium-droplet state on (Figure 6a) alternates with the state on (Figure 6b) in which neutron scattering is not observed - the Bragg's peaks are missing, which means that there are no vortices and anti-vortices. The X-ray diffraction from helium nanodroplets (panel b) shows that: the droplet in state b was elongated toward the poles of the torus, which can be seen by the more spaced diffraction lines at the poles, and energy was emitted from them; at the same time, the droplet shrunk at the equator, which can be seen from more narrowly spaced diffraction lines in direction equator.

At the end of her article, when trying to explain the lack of Bragg's peaks on (Figure 6b) which means lack of vortices and antivortices, Ashley Smart cited the authors' opinion [10] that "this could be a sign of new physics". This "new physics" is the already developed branch of physics, called nonlinear physics, which describes dynamic systems.

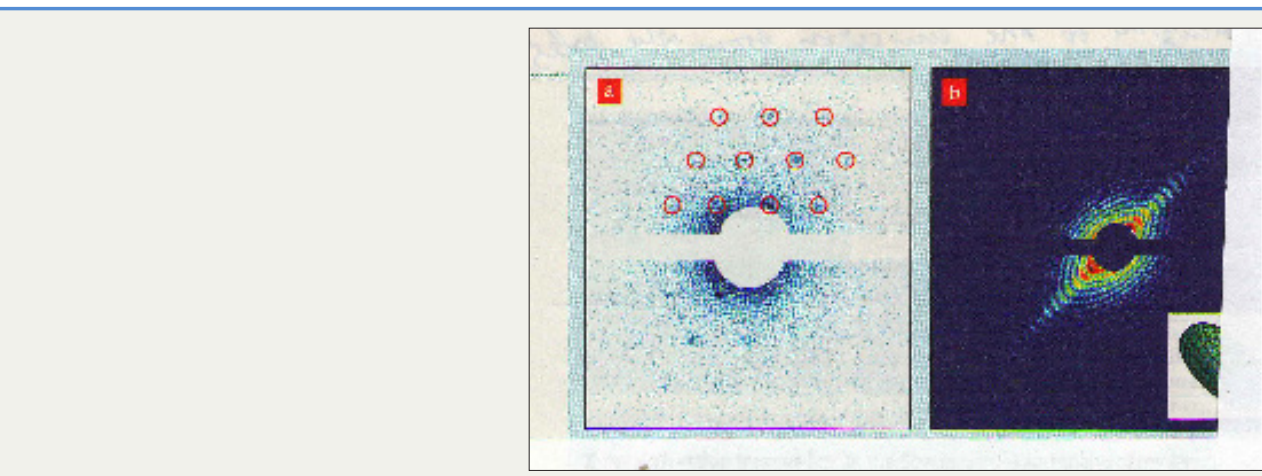

Figure 6:

6a: Bragg's lattice from a droplet bulged at the equator with quantized vortices in the equatorial area.

6b: A droplet with no Bragg's scattering, which means vortices are no longer present. X-ray diffraction shows a shrunk equatorial area and elongation along the poles emitting energy. 
In our book [4], a dynamic model was offered showing that Bragg's peaks were not observed in state $b$ (Panel b) because the droplet became elongated toward the poles, emitted some energy through the poles, and its turbulence seized, i.e. the vortices and anti-vortices disappeared.

It is easy to understand the misconception of the authors of article [9], who wanted to see the droplet in state b the way it was (flattened at the poles and bulged at the equator). However, the fact that no vortices were observed means that something dramatic has happened and my model [12] shows and (Panel b) confirms, that the droplet became elongated toward the poles, energy was emitted through them, and the turbulence in the equatorial area seized to exist.

Our Sun has the donut-shape NEMF of the helium nano-droplets and it has the same dynamic. During each active cycle our Sun spins faster is belched at the equator and has a lot of turbulence at the equators observed as solar spots. When a critical level of pressure from the belching at the equator is reached, which the solar plasma can tolerate (at a critical speed of spinning), the sun shifts to another shape. It becomes elongated at the poles energy is emitted through the poles and the solar activity seizes because solar spots are not observed which means there is no turbulence [16].

\section{Quantum Energy Levels and Torus-shaped NEMF-How Common are They?}

Not only are the NEMF of humans, Sun, and Earth torus-shaped, such is the NEMF of helium nanodroplets $[10,15]$. Even elementary particles seems to have the same donut shape EMF with the same dynamic. The elementary particles emit from their equatorial area virtual photons and swallow them back just like our Sun emits spinning energy balls from its anti-vortices, which after a loop trajectory are swallowed back by adjacent vortices.

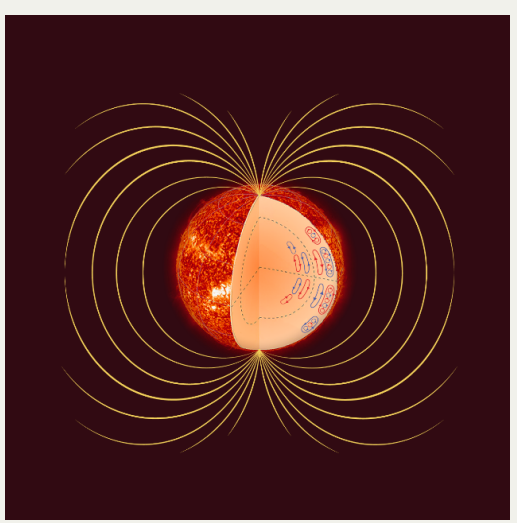

Figure 7: Sun's torus-shaped nonlinear EMF (vertical cross-section). Visible are the ring currents induced by the sun's spinning, which crank the magnetic field.

Not only are the energy levels of elementary particles, atoms, and molecules discrete or quantized, looking at the radiation belts of our Earth we see discrete levels and looking at our Sun we see discrete orbits. Even the rings of Saturn are discrete. The same is the situation with the human NEMF- our energy levels are discrete
(Figure 7 \& 8) [5].

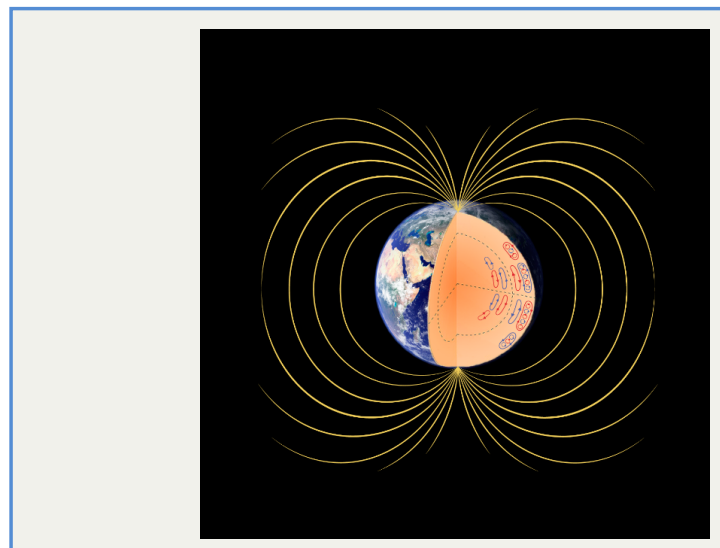

Figure 8: Earth's torus-shaped nonlinear EMF (vertical cross-section). Visible are the ring currents induced by the earth's spinning, which crank the magnetic field.

The dynamic of helium nanodroplets seems to be alternating shifts between two shapes:

i) The shape of a torus when the nanodroplets spin fast around axis passing through the hole of the donut is bulged at the equator, and exhibit turbulence with observed Bragg's neutron scattering

ii) A state of low activity at the equator, when Bragg's neutron scattering is not observed. X-rays diffraction revealed that the nanodroplets are shrunk at the equator, elongated toward the poles, and emitting energy from both ends of the axis of spinning [5].

What caused the shift to the second state? It seems that as the helium droplet was spinning faster and faster, the bulging at the equator reached the critical value of pressure which the helium substance can tolerate. To release the pressure the droplets started spinning in opposite direction the magnetic poles of the donut distanced each other, energy was emitted through the poles (observed with X-ray diffraction), and the turbulent activity in the equatorial area seized (Bragg's neutron scattering was not observed).

\section{Conclusion}

Nonlinear physics needs to step in to finish the picture of the dynamic. During the active cycle, the helium nanodroplets are torus-shaped, spin faster and faster, suck energy through the poles, bulge at the equator, and exhibit turbulence in the equatorial area. For this to happen, the donut needs to spin clockwise like a vortex. Then both magnetic poles (of the induced by the spinning EMF) would be vortices sucking energy in because the torus shape results from the superposition of vortex on top of anti-vortex $[4,6]$.

When the bulging at the equator reaches the critical value of pressure, which the substance of the helium nanodroplets can tolerate, to release the pressure the spinning must switch from clockwise to counterclockwise. Then the two magnetic poles would be anti-vortices emitting energy. Indeed, the X-rays diffraction from 
the helium nanodroplets revealed emission of energy from both poles, which ended the turbulent activity.

This dynamic seems to be typical for all torus-shaped electromagnetic fields (EMF) including our Sun [16,17]. The alternative switches between high solar activity (warm periods on Earth) and low solar activity (Ice Ages on Earth) must be a product of these switches from clockwise spinning of the Sun during the warm periods to counterclockwise spinning during the Ice Ages.

The astronomers speak about this as periodic reversal of the magnetic polarity of the Sun. However, this actually means periodic reversal of the direction of spinning of the Sun. When the Sun spin clockwise, it sucks energy in, spins faster and faster, becomes bulged at the equator, and exhibit turbulence in the equatorial area seen as solar spots. This is the period of high solar activity.

When the bulging at the equator reaches the critical value of pressure, which the solar plasma can tolerate, to release the pressure the sun stars spinning in opposite (counterclockwise) direction. The two magnetic poles start emitting energy and distance each other. The sun shrinks at the equator and its turbulent activity ends. This continues until the pressure at the equator reaches a critical value. To release the pressure, the sun will start spinning in opposite (clockwise) direction, suck energy, and become active again, and this goes on and on.

\section{References}

1. Prigogine I (1990) Time, dynamics, and chaos. University of Texas, USA.
2. https://www.heartmath.com/

3. Kuman M (2018) New light on the attractors creating order out of the chaos. Int J Complement Alt Med 11(6): 337-342.

4. Kuman M (2017) Same fields and dynamics in stars and man. Health and Happiness Books.

5. Tichoplav V, Tichoplav T (2012) Miroznanie world view. Saint Petersburg, Russia.

6. Kuman M (2015) Quantum mind and quantum growth. Health and Happiness Books.

7. Kuman M (2005) Science speaks of god. Health \& Happiness Books.

8. Williamson SJ (1989) Advances in biomagnetism, Plenum Press, Berlin.

9. Nance S (2017) Monthly notices of the royal astronomical society 453(3): 2654-2661.

10. Gomez LF (2014) Science 45: 906.

11. Kirlian SD (1949) Method of photographing different objects patent N106401 USSR, Moscow, Russia.

12. Vasilatos G (1999) Lost Science. Adventures Unlimited Press, USA.

13. Burgay M (2004) Nature 426: 531-533.

14. Lyne AG (2004) Science.

15. Smart A (2014) Physics Today 67: 11-16.

16. Kuman M (2016) Dynamic mathematical model describes the dynamic of solar activity APS meeting in Maryland, US.

17. Y Manaka (1995) Chasing the dragon's tail. Paradigm Publications, USA.
Creative Commons Attribution 4.0 International License

For possible submissions Click Here

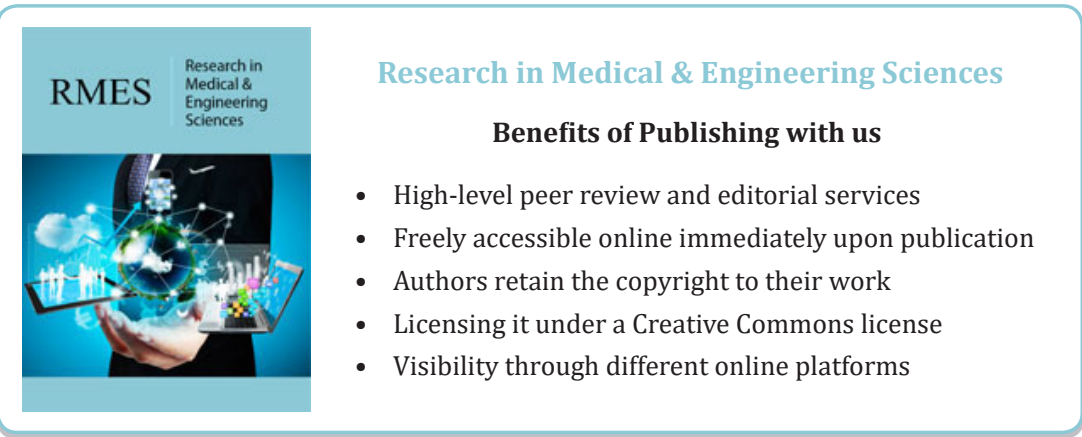

